\title{
Relatos médicos sobre formação acadêmica e trabalho na Estratégia de Saúde da Família
}

\author{
Medical reports on academic training and work in Family Health Strategy \\ Informes médicos acerca de la educación y trabajo en la Estrategia de Salud de la Familia \\ Jaqueline Santos Barboza, Amanda Batista da Silva Lemos, Bruna Fraga Guimarães Barreiro, Gabriela Bernardes \\ Martins Peixoto
}

\section{Resumo}

Objetivo: Analisar as percepções dos médicos atuantes na Estratégia de Saúde da Família de um município de médio porte no estado de Minas Gerais sobre a formação e atuação neste nível de atenção. Métodos: Trata-se de uma pesquisa de abordagem qualitativa, exploratória e descritiva, que avaliou o universo de significados, crenças, valores e atitudes dos participantes em relação ao tema. Os dados foram coletados mediante entrevista semiestruturada. Resultados: Foram realizadas 12 entrevistas com médicos de 11 unidades de saúde. Os sujeitos entrevistados relatam que os conhecimentos referentes à Estratégia de Saúde da Família no currículo da graduação foram satisfatórios e contribuíram com sua formação e atuação profissional. No entanto, a escolha pelo trabalho ainda aparece como um caminho facilitador para entrada na residência médica, principalmente por meio de programas governamentais. Conclusões: Ainda que a pesquisa tenha abordado os relatos de uma pequena parcela de médicos, a análise evidencia a importância da educação médica na sensibilização e capacitação em medicina de família e comunidade, bem como a ainda necessária valorização da carreira no nível primário de atenção. Estas temáticas permanecem no bojo das discussões quando se trata da consolidação desta estratégia no país.

\section{Abstract}

Objective:To analyze the perception of physicians working in the Family Health Strategy of a medium-sized municipality in the state of Minas Gerais, about the training and work in this field of activity. Methods: This is a qualitative, exploratory and descriptive research, which addressed the universe of meanings, beliefs, values and attitudes of the participants towards the subject. Results: 12 interviews were conducted with physicians from 11 health units. The subjects interviewed report that the knowledge referring to the Family Health Strategy in their undergraduate curriculum was satisfactory and contributed to their professional training and performance. However, the choice of work still appears as a facilitating pathway to medical residency, mainly through government programs. Conclusions: Although the research has addressed the reports of a small number of physicians, the analysis highlights the importance of medical education in sensitizing and training for family and community medicine, as well as the still needed career enhancement in the primary care level. These topics remain in the heart of the discussions when it comes to the consolidation of this strategy in the country.

\section{Palavras-chave:}

Medicina de Família e Comunidade

Educação Médica

Estratégia de Saúde da Família

\section{Keywords:}

Community Family Practice

Medical Education

Family Health Strategy
Como citar: Barboza JS, Lemos ABS, Barreiro BFG, Peixoto GBM. Relatos médicos sobre formação acadêmica e trabalho na Estratégia de Saúde da Família. Rev Bras Med Fam Comunidade. 2016;11(38):1-8. http://dx.doi.org/10.5712/rbmfc11(38)1393
Fonte de financiamento: declaram não haver. Parecer CEP: 1.070.008 (UNIFENAS), aprovado em 20/05/2015. Conflito de interesses: declaram não haver. Procedência e revisão por pares: revisado por pares. Recebido em: 11/09/2016. Aprovado em: 15/11/2016. 


\section{Resumen}

Objetivo: Analizar las percepciones de los médicos que trabajan en la Estrategia de Salud de la Familia de un municipio de tamaño medio en el estado de Minas Gerais, en la formación y trabajo en este nivel de atención. Métodos: Se trata de una investigación cualitativa, exploratoria y descriptiva, que abordó el universo de significados, creencias, valores y actitudes de los participantes en el tema. Resultados: Se realizaron 12 entrevistas con médicos de 11 unidades de salud. Los entrevistados informan que el conocimiento relativo a la Estrategia de Salud de la Familia en el plan de estudios de licenciatura fue satisfactorio y contribuyó a su formación y desempeño profesional. Sin embargo, la elección del trabajo continúa apareciendo como una forma facilitadora para la entrada en la residencia médica, principalmente a través de los programas de gobierno. Conclusiones: Aunque la investigación se ha ocupado de los informes de un pequeño porcentaje de los médicos, el análisis pone de relieve la importancia de la educación médica en la sensibilización y la formación en medicina familiar y comunitaria, así como la mejoría de la carrera sigue siendo necesaria a nivel de atención primaria, cuestiones que quedan en medio de discusiones cuando se trata de la consolidación de esta estrategia en el país.
Palabras clave:

Medicina Familiar

y Comunitaria

Educación Médica

Estrategia de Salud Familiar

\section{Introdução}

Neste trabalho apresentamos uma interlocução entre a formação acadêmica e a atuação médica na Atenção Primária à Saúde (APS), por meio da análise dos relatos dos médicos que atuam na Estratégia de Saúde da Família (ESF). A importância da atuação médica no fortalecimento do sistema e no adequado atendimento à população justificou esse trabalho, que buscou delimitar o perfil de graduação de médicos num município de médio porte do Sul de Minas Gerais.

No Brasil, o Sistema Único de Saúde (SUS) absorve uma grande parcela de médicos, especialmente na ESF, porta de entrada comum para o mercado de trabalho. Apesar de o médico representar um elemento fundamental nas equipes de saúde da família, algumas barreiras são encontradas na execução de seu trabalho, supostamente calcadas na deficiência curricular em saúde coletiva.

É consensual que a consolidação da ESF depende da efetiva qualificação do profissional de saúde, em especial do médico, que neste contexto não pode se limitar a um atendimento curativo em função de uma emergência qualquer. É preciso integrar-se à equipe com o intuito de tornar sua função proativa: atuando na prevenção de doenças e na promoção da saúde comunitária por meio de atividades comunicativas e das ações técnicas mais diversas. ${ }^{1}$

Elemento essencial nas equipes mínimas de Saúde da Família, os médicos compõem um conjunto de agentes capazes de desenvolver ações de saúde, sendo, deste modo, uma importante força de trabalho em saúde. ${ }^{2}$ Portanto, é fundamental que o processo de formação acadêmica e profissional seja capaz de formar profissionais que atuem nos diferentes níveis de atenção à saúde, em especial nos programas que requerem ações de promoção, prevenção, recuperação e reabilitação da saúde, nos âmbitos individual e coletivo. ${ }^{3}$

É possível que o ensino na comunidade, adotando a ESF como locus de aprendizagem da educação médica, propicie aos médicos recém-formados maior segurança e preparação para atuar na ESF, ao contrário dos profissionais formados em currículos antigos. ${ }^{4}$

Neste sentido, as Diretrizes Curriculares dos Cursos de Medicina $^{3}$ enfatizam a necessidade de formar um profissional adequado às necessidades do sistema de saúde pública, além de refletirem um cenário de transição e consolidação de um novo modelo assistencial em saúde no Brasil que preconiza e orienta a organização dos cursos de medicina, de forma a estimular a formação do médico comprometido com as demandas do Sistema Único de Saúde, o SUS. 
As reformas no plano curricular nos cursos de graduação em saúde projetaram estratégias que permitem ao aluno experienciar a Atenção Primária em seu contexto mais amplo pela influência direta com os usuários do sistema de saúde, com as equipes de Saúde da Família e nos locais onde essa prática se desenvolve. Isso impulsiona as bases que possibilitam ao estudante de Medicina captar o sentido de toda a formação médica. ${ }^{5}$

\section{Métodos}

Tratou-se, portanto, de uma pesquisa de abordagem qualitativa, exploratória e descritiva, que abordou o universo de significados, crenças, valores e atitudes relacionadas ao tema. ${ }^{6}$

A coleta de dados ocorreu por meio de entrevista semiestruturada, que permite maior flexibilidade na sua condução e ao mesmo tempo um levantamento de dados objetivos. As questões abordadas na entrevista enfatizaram a percepção dos entrevistados sobre sua própria formação acadêmica e sobre o seu trabalho na ESF.

O roteiro continha questões como: presença de conteúdos sobre ESF/APS na graduação, suficiência da formação na área, possíveis falhas curriculares e discrepâncias entre graduação e prática. Questionou-se, também, as facilidades e dificuldades no trabalho na APS, a escolha do campo de trabalho e a percepção pessoal sobre atuação na ESF.

As entrevistas, que aconteceram em Unidades de Saúde da Família (USF) do município, durante o turno de trabalho habitual dos profissionais e mediante prévia autorização dos gestores da Secretaria Municipal de Saúde do município, foram individuais e sigilosas, em ambiente reservado para tal.

As entrevistas foram gravadas e os dados passaram (1) organização dos relatos em uma ordem, criando subconjuntos para leitura das homogeneidades e das diferenciações; (2) elaboração transversal do conjunto ou de cada subconjunto do material empírico, com a intenção de organizar tecnicamente subconjuntos separados por assuntos, constituindo já a primeira forma de classificação do material; (3) o material contido nos muitos subconjuntos passou por uma nova leitura e organização, re-arrumado em tópicos que os entrevistados destacaram, sobretudo, por meio da reiteração. A interpretação deste produto final foi feita, sobretudo, por meio do método comparativo, por categorias de análise que emergiram da literatura analisada na revisão da literatura. ${ }^{7}$

Os participantes foram orientados quanto aos objetivos da pesquisa e formalizaram a participação mediante Termo de Consentimento Livre e Esclarecido. A pesquisa teve aprovação do Comitê de Ética em Pesquisa, sob o parecer número 1.070.008.

\section{Resultados e Discussão}

Foram realizadas 12 entrevistas com médicos de 11 Unidades de Saúde da Família (USF). Apenas um médico de uma unidade de saúde especifica se recusou a participar da presente pesquisa. Considerando que a cidade possui 12 Unidades de ESF, essa amostra corresponde a aproximadamente $92 \%$. A grande maioria dos entrevistados (75\%) tem formação acadêmica em um mesmo campus de uma mesma universidade, e $67 \%$ desses médicos possuem tempo de trabalho na ESF menor que 4 anos, sugerindo, portanto, formação acadêmica recente. 


\section{APS/ESF no currículo da graduação médica}

Dos médicos entrevistados, todos relataram contato com conteúdos teóricos e práticos da APS na graduação, incluindo procedimentos básicos como aferir pressão, triagem e visitas domiciliares. Além disso, relataram que houve o contato com a estrutura das Unidades de Saúde do primeiro ao último período, em formato de disciplinas práticas, estágios e módulos profissionalizantes.

“Sim, na faculdade desde o primeiro ano, eles já estão fazendo a formação mais voltada, né?, para o atendimento básico, atendimento primário de PSF. Então, desde o primeiro período de faculdade a gente teve tanto a parte prática, onde a gente já tinha contato com o PSF, acompanhava, via a estrutura e tudo. Na parte teórica também, a gente tinha umas aulas voltadas... Então já teve como ter uma base de como é que seria esse trabalho no PSF." (M3)

Este resultado difere do encontrado por Gonçalves et al., ${ }^{8} \mathrm{em}$ um estudo semelhante com os médicos atuantes na ESF da cidade de Botucatu (SP) entre os anos 2006 e 2007, cujo resultado havia sido unânime em referência ao curso de graduação não ter dado destaque e capacitação aos médicos para escolher e exercer a profissão na APS.

A diferença entre os resultados pode ser atribuída pela consolidação, ainda que tardia, das Diretrizes Curriculares Nacionais do Curso de Graduação em Medicina, que no ano de 2001 propunham o aprendizado por competências e a formação de um egresso com compreensão ampliada de saúde e consciência da realidade em que atua. A integralidade constituía um dos principais elementos de um currículo baseado em competências, agregado ao Sistema de Saúde (SUS) e à universidade, numa formação em que a Atenção Primária a Saúde é evidenciada como norteadora dessa prática integral. ${ }^{9}$

As novas diretrizes, aprovadas em 2014, continuaram a incorporar novos avanços na educação médica. Um desses avanços foi a inserção de estágio curricular obrigatório de formação em serviço, em regime de internato, com carga horária mínima de 35\% da carga horária total do curso de Medicina. Dessa carga horária mínima, 30\% deverá ser desenvolvida na Atenção Básica e em Serviços de Urgência e Emergência do SUS com predomínio dos serviços de Atenção Básica. Esses serviços ainda devem ser coordenados e voltados para a área de Medicina Geral de Família e Comunidade. ${ }^{3}$

Os médicos entrevistados consideraram-se suficientemente preparados pela graduação para atuação na ESF, ainda que alguns destaquem certa sensação de insegurança nos primeiros dias de trabalho. Segundo eles, a existência de estágios na APS durante o curso complementou de forma satisfatória a formação, capacitando-os para exercer sua profissão na ESF.

“(...) Então, nós sentimos na realidade o que é ser médico de família, o que, como que a gente poderia atuar e me deu uma base muito boa para o que eu sou hoje dentro da saúde da família." (M6)

Os entrevistados apontaram, ainda, níveis crescentes de complexidade dos procedimentos realizados na APS no decorrer da graduação. De fato, as Diretrizes Nacionais do Curso de Medicina de 2001 estabeleciam que na formação médica o estudante deve ser capacitado a atuar nos diferentes níveis de atendimento à saúde, assim como de utilizar adequadamente os recursos semiológicos e terapêuticos para cada um desses níveis de atenção. ${ }^{9}$ 
"À medida que a gente avançava de período nossas atividades em relação à atenção primária se tornavam mais complexas, [...] a partir do quarto ano já atendíamos os pacientes." (M1)

A maior parte dos entrevistados afirmou que quanto mais o ensino acadêmico priorizar a realização de atividades práticas na Atenção Primária, melhor será para a formação do médico. Além disso, entre as sugestões dos entrevistados para melhorar a qualidade dos atendimentos na ESF, a principal foi intensificar as atividades práticas na disciplina de APS, podendo, por exemplo, mesclar teoria e prática já nos primeiros anos. Em síntese, a maioria dos entrevistados indicou um enfoque curricular na ESF por parte da universidade.

"Eu acho que quanto mais prática, mais você vai ter facilidade na hora de trabalhar sozinho... Porque você vai aprender na prática, vai colocar o que aprende em sala de aula aqui (no PSF)". (M7)

"Saber diferenciar e dar mais um pouquinho de importância para a preparação clínica do médico que tem interesse em PSF. Porque eu acho que de forma geral nós somos preparados como generalistas, mas esta visão de que o médico do PSF é um clínico diferente fica às vezes um pouquinho a desejar [...]." (M12)

"A gente percebe uma tendência, até mesmo no ponto de vista do próprio governo,de uma correção de grade para focar mais a atenção primária." (M10)

De modo similar, as diretrizes Curriculares Nacionais dos Cursos de Graduação em Medicina enfatizam a necessidade de formar um profissional adequado às necessidades sociais da saúde com ênfase no SUS por intermédio dessa integração ensino-serviço como um espaço de ensino-aprendizagem. As necessidades dos pacientes e da comunidade em geral são norteadoras de uma possível transformação social. Aproximar a formação do médico das realidades de saúde e de trabalho, segundo as Diretrizes Curriculares Nacionais do curso, tende a formar um profissional competente, ético e comprometido com as necessidades de saúde da população. ${ }^{3,4}$

\section{O trabalho na APS/ESF: complexidade e desafios}

Apesar de a ESF ser um serviço de baixa densidade tecnológica, suas equipes devem ser capazes de resolver $85 \%$ dos problemas de saúde em sua comunidade, prestando atendimento de bom nível, prevenindo doenças, evitando internações desnecessárias e melhorando a qualidade de vida da população. ${ }^{10}$ O trabalho do médico de Família e Comunidade é bastante complexo, e é essencial o domínio de um grande espectro de conhecimentos, bem como habilidades e atitudes para enfrentar a complexa realidade biopsicossocial dos pacientes, família e comunidade. ${ }^{11}$

De acordo com Rosa \& Labate, ${ }^{12}$ a ESF introduz uma nova visão sobre o trabalho em saúde ao colocar a família e a saúde como centros de atenção (e não somente o indivíduo ou a doença), e buscar um modelo de atenção que age de forma preventiva. De fato, a maioria dos médicos entrevistados relataram que o trabalho médico na ESF fundamenta-se em promoção de saúde, ou seja, prevenir doenças e "ver o paciente como um todo", extrapolando a esfera curativa. 
“Trabalhar numa ESF é justamente a questão de olhar o paciente de uma forma mais abrangente." (M2)

O trabalho médico na ESF excede a esfera da intervenção curativa individual e inclui, principalmente, demandas sanitárias que geram as ações tradicionais da saúde pública (saneamento do meio, desenvolvimento nutricional, a vacinação ou a informação em saúde), as demandas relacionadas a ações clínicas (prevenção, profilaxia e o tratamento de doenças de caráter epidêmico). ${ }^{12}$

"Você conseguir cuidar da saúde desses pacientes de forma preventiva, lá na frente o índice de doença vai ser muito menor." (M7)

Entretanto, parte disso não parece ser concretizado na prática, fato que a maior parte dos entrevistados aponta como a discrepância entre teoria e prática, justificada, principalmente, pela impossibilidade de se executar nas Unidades de Saúde algumas práticas e diretrizes vistos na teoria.

"É, nada acontece como a teoria né? (...) Mas a gente se vê meio que obrigado a fazer conforme o serviço aqui funciona, a gente não pode agir conforme o que a gente aprende só." (M4)

"Boa parte do conteúdo não é possível aplicar na prática, (...) como por exemplo a consulta programada, isso não funciona, infelizmente, na prática. O material que se tem disponível para trabalhar é complicado. A gente poderia fazer muito mais coisa na atenção básica para melhorar o atendimento." (M1)

As dificuldades de aplicar na prática aquilo que é ensinado na teoria aos médicos foram atribuídas à falta de tempo, de infraestrutura, organização do serviço e excesso de demanda, necessitando, por essa razão, adaptarem suas atividades práticas de acordo com o que é possível.

"Se funcionasse realmente como está nas regras, no edital, no próprio SUS... seria tudo lindo, né?" (M3)

A complexidade do trabalho na ESF pode ser relacionada com sua responsabilidade na resolução dos problemas de saúde da comunidade. Já os desafios do trabalho na APS podem ser associados à discrepância entre teoria e prática, e devido à falta de investimentos governamentais para organização plena do serviço.

O desafio da valorização da carreira

Sabe-se que a ESF ocupa lugar de transição na carreira médica, como uma etapa entre a graduação e a especialidade ou o sistema privado. De fato, grande parte dos médicos entrevistados relataram que trabalhar na ESF é um facilitador para entrada em programas de Residência Médica, especialmente se ligados à programas que pontuam o currículo (como PROVAB, por exemplo). Um pequeno grupo afirmou que a escolha pela ESF foi devido a sua importância social, enquanto muitos justificaram a escolha com base na necessidade financeira e as poucas opções para um médico recém-formado.

"Escolhi para contar pontos para residência porque faço parte do programa do PROVAB e também porque ainda não sei no que se especializar para fazer a residência." (M8) 
Nogueira ${ }^{1}$ afirma que a falta de valorização e reconhecimento para os médicos dessa área são apontados como fatores que colaboram para a alta rotatividade dos médicos da ESF, e a inexistência de um plano de carreira para o médico na Saúde Pública contribui para esta realidade, como se observam nos relatos:

"Esse ano sim, porque eu não sei o que escolher na residência não. Mas eu não quero seguir com o PSF pro resto da minha vida não." (M1)

"Não, eu digo que pra trabalhar em PSF deveria ser um médico com residência em PSF, eu acho que é muito triste você colocar um recém-formado num PSF. A gente, aqui a gente pega de tudo, então aqui eu tenho que mexer com ortopedia, urologia, nefro, GO, pediatria, todas as especialidades e a gente tem uma noção durante a faculdade, né? (...)" (M4)

Dessa maneira, ainda há um nítido descompasso entre a retórica oficial, que atribui grande importância à Saúde da Família, e as medidas efetivas de formação e regulação da especialidade no país. ${ }^{13}$ Portanto, far-se-ia necessário principalmente, a criação de um plano de carreira para essa área para maior motivação do médicos para atuação na área.

\section{Considerações Finais}

Os sujeitos entrevistados relatam que os conhecimentos referentes à ESF no currículo da graduação foram satisfatórios e contribuíram com sua formação e atuação profissional, de modo que se pode destacar o papel fundamental do currículo da graduação médica na qualificação profissional dos médicos que atuarão em medicina de família e comunidade.

No entanto, a escolha pelo trabalho na ESF ainda aparece como um caminho facilitador para entrada na residência médica, principalmente por meio de programas governamentais, o que dificulta a permanência desses médicos no local, inviabilizando a continuidade da atenção (longitudinalidade) e a integração com a comunidade.

Ainda que esta pesquisa tenha abordado o universo de uma pequena parcela de médicos atuantes na ESF, a análise dos relatos evidencia a importância da educação médica na sensibilização e capacitação em medicina de família e comunidade, bem como a ainda necessária valorização da carreira no nível primário de atenção, temáticas que permanecem no bojo das discussões quando se trata da consolidação da ESF no país.

\section{Referências}

1. Nogueira RP. A questão do médico em saúde da família. Rev Desafios do Desenvolvimento. 2006; 28(3). [Citado 2016 Dez 15]. Disponível em: http://www.ipea.gov.br/desafios/index.php?option=com_content\&view=article\&id=853:catid=28 \&ltemid $=23$

2. Feuerwerker LCM. Além do discurso de mudança na educação médica: processos e resultados. Rio de Janeiro: Hucitec; 2002.

3. Brasil. Ministério da Educação. Conselho Nacional de Educação. Câmara de Educação Superior. Resolução no 3, de 20 de junho de 2014. Diretrizes Curriculares Nacionais do Curso de Graduação em Medicina e outras providências. Brasília: Ministério da Educação; 2014. 
4. Nóbrega-Therrien SM, Souza PMM, Pinheiro FMC, Castro VS. Formação para estratégia saúde da família na graduação em medicina. Rev Bras de Educ Med. 2015;39(1):112-8. DOI: http://dx.doi.org/10.1590/1981-52712015v39n1e02212012

5. Souza MB, Rocha PM, Sá AB, Uchoa SAC. Trabalho em equipe na atenção primária: a experiência de Portugal. Rev Panam Salud Publica. 2013;33(3):190-5. DOI: http://dx.doi.org/10.1590/S1020-49892013000300005

6. Minayo MCS, org. Pesquisa social: teoria, método e criatividade. 28를 ed. Petrópolis: Vozes; 2009.

7. Minayo MCS. O desafio do conhecimento: pesquisa qualitativa em saúde. 14ª ed. São Paulo: Hucitec; 2014.

8. Gonçalves, RJ, Soares RA, Troll T, Cyrino EG. Ser médico no PSF: formação acadêmica, perspectivas e trabalho cotidiano. Rev Bras Educ Med. 2009;33(3):382-92. DOI: http://dx.doi.org/10.1590/S0100-55022009000300009

9. Brasil. Ministério da Educação. Conselho Nacional de Educação. Câmara de Educação Superior. Resolução CNE/CES no 4, de 7 de novembro de 2001. Institui Diretrizes Curriculares Nacionais do Curso de Graduação em Medicina. Brasília: Diário Oficial da União; 2001.

10. Brasil. Ministério da Saúde. Sistema Único de Saúde (SUS): princípios e conquistas. Brasília: Ministério da Saúde; 2000. 41 p.

11. Falk JW. A Medicina de Família e Comunidade e sua entidade nacional: histórico e perspectivas. Rev Bras Med Fam Comunidade. 2004;1(1):5-10. DOI: http://dx.doi.org/10.5712/rbmfc1(1)2

12. Rosa WAG, Labate RC. Programa Saúde da Família: a construção de um novo modelo de assistência. Rev Latino-Am Enfermagem. 2014;13(6):1027-34. DOI: http://dx.doi.org/10.1590/S0104-11692005000600016

13. Rodrigues PHA, Ney MS, Paiva CHA, Souza LMBM. Regulação do trabalho médico no Brasil: impactos na Estratégia de Saúde da Família. Physis. 2013;23 (4):1147-66. DOI: http://dx.doi.org/10.1590/S0103-73312013000400007

Jaqueline Santos Barboza. Universidade José do Rosário Vellao (UNIFENAS). Alfenas, MG, Brasil. jaquelinesb_psi@ hotmail.com (Autora correspondente)

Amanda Batista da Silva Lemos. Universidade José do Rosário Vellao (UNIFENAS). Alfenas, MG, Brasil. mandinhah_1995@ hotmail.com

Bruna Fraga Guimarães Barreiro. Universidade José do Rosário Vellao (UNIFENAS). Alfenas, MG, Brasil. brunarbdb@ hotmail.com

Gabriela Bernardes Martins Peixoto. Universidade José do Rosário Vellao (UNIFENAS). Alfenas, MG, Brasil. gabibmp@ hotmail.com 